\title{
Labyrinthe
}

20 | 2005 (1)

La Cognition

\section{Les pachas du sultan}

Essai prosopographique sur les agents supérieurs de l'État ottoman

(1839-1909)

\section{Olivier Bouquet}

\section{(2) OpenEdition}

1 Journals

Édition électronique

URL : http://journals.openedition.org/labyrinthe/767

DOI : $10.4000 /$ labyrinthe.767

ISSN : 1950-6031

Éditeur

Hermann

Édition imprimée

Date de publication : 20 avril 2005

Pagination : 157-160

Référence électronique

Olivier Bouquet, « Les pachas du sultan », Labyrinthe [En ligne], 20 | 2005 (1), mis en ligne le 09 juillet 2008, consulté le 01 mai 2019. URL : http://journals.openedition.org/labyrinthe/767 ; DOI : 10.4000/ labyrinthe.767

Propriété intellectuelle 


\title{
LES PACHAS DU SULTAN \\ Essai prosopographique sur les agents supérieurs de l'État ottoman (1839-1909)*
}

\author{
Olivier BOUQUET \\ olbouquet@voila.fr
}

Au-delà de la figure savoureuse d'une sorte de Turc ventripotent et avachi au milieu de son harem ou d'un janissaire sanguinaire croqué dans les récits de voyageurs européens et dans certaines sources littéraires ottomanes, les pachas sont des agents de l'État honorés par le sultan du plus prestigieux des titres de la fin de l'Empire. Comme ils accèdent à cette dignité lorsqu'ils sont promus aux grades supérieurs de l'administration civile et militaire ${ }^{1}$, aucun d'entre eux n'est religieux; les rares oulémas qui le deviennent se trouvent dans l'obligation d'échanger leur turban contre le fez du bureaucrate. En leur qualité de hauts dignitaires, les pachas sont - pour un grand nombre d'entre eux répertoriés parmi des dizaines de milliers de membres de l'administration civile dans les dossiers de personnel (sicill-i ahval) des archives de la présidence du Conseil d'Istanbul. Constitués sous le règne d'Abdülhamid II (1876-1909), ces registres regroupent les notices biographiques des agents en fonction tout au long du XIX ${ }^{\mathrm{e}}$ siècle, enregistrées sur un mode systématique sous des rubriques aussi diverses que l'origine familiale, sociale et géographique, la formation scolaire et professionnelle, les langues pratiquées, les postes successifs occupés, les salaires perçus, le rang et le mérite, et les décorations reçues.

La qualité et la richesse de la source permettent l'exploitation sérielle d'un échantillon de près de trois cents de ces notices. Elles

\footnotetext{
* Thèse de doctorat en histoire soutenue le 17 décembre 2004 à l'EHESS, sous la direction de M. Gilles Veinstein, directeur d'études à l'EHESS et professeur au Collège de France.

1. Cette attribution est implicative, pour reprendre le terme de Luc Boltanski, « L'espace positionnel. Multiplicité des positions institutionnelles et habitus de classe », Revue française de sociologie, vol. XIV, 1973, p. 4
} 
offrent l'intérêt d'aborder en profondeur une archive particulièrement négligée jusqu'à présent, d'en décrire la genèse administrative et d'en établir l'utilité pour l'historien. Elles conduisent à rapprocher une opération administrative d'envergure et la naissance du genre autobiographique chez les derniers Ottomans : la formulation biographique, par le récit, du rapport de l'individu isolé face à l'État, semble en effet participer à l'affirmation littéraire du souci de soi. Elles invitent à considérer ensemble deux périodes de la réforme ottomane (les Tanzimat de 1839 à 1876 et la période hamidienne) généralement dissociées par l'historiographie. Enfin, elles permettent de proposer une étude détaillée des groupes qui assurent le fonctionnement de l'État ottoman. Dans cette perspective, un catalogue de notices est d'abord conçu afin de réorganiser les données de la source selon des rubriques systématiques, tout en proposant pour chaque pacha un récit analytique de la carrière.

L'exploitation de ce catalogue conduit dans un second temps à la mise en œuvre d'une prosopographie (considérée dans ce travail aussi bien comme biographie collective que comme histoire sociale des institutions), par le moyen d'une analyse qui emprunte successivement trois directions. Une étude des caractéristiques principales des pachas (grades, traitements, fonctions, décorations) est d'abord proposée. Elle révèle que le monde des pachas est un lieu d'usages établis et distincts, que la carrière obéit à des lignes régulières de progression qui s'imposent à tous, et que le favoritisme et les logiques de passe-droits, loin d'abolir les règles de progression, ne font que les contourner par endroits. D'un côté, les pachas sont des hommes à tout faire issus de domaines d'activité variés, mais qui ont en commun d'exercer des fonctions parmi les plus élevées de l'État. De l'autre, le titre renvoie à des fonctions, des carrières, des niveaux de traitement, des plastrons, des rapports au sultan si divers qu'il n'est pas étonnant que les Ottomans n'y voient pas un groupe collectif et que les pachas eux-mêmes ne se soient pas identifiés à un corps.

La recherche propose ensuite une analyse des conditions qui fait retour sur les rythmes divers et enchevêtrés de la socialisation ottomane et aborde les individus comme les produits d'une fabrique sociale, scolaire et linguistique. Les pachas sont ici envisagés aussi bien comme serviteurs du sultan que comme sujets d'un empire: administrés avant d'être administrateurs, ils ont tous vécu dans le même monde, ont 
fréquenté des écoles semblables, se sont inclinés au passage des grands et des illustres, ont reconnu la même autorité politique. Mais dans cette fabrique, ils ont été façonnés dans un atelier particulier: plus d'un pacha sur cinq est fils de pacha; plus d'un pacha sur quatre est fils ou descendant de pacha. Néanmoins, l'hérédité du titre s'épuise généralement au bout d'une génération, en sorte qu'il convient mieux de parler d'une noblesse dans l'État que d'une noblesse d'État. En outre, les pachas ont été bien plus scolarisés que la plupart des Ottomans. Ce qui les différencie vis-à-vis du reste de la société est considérablement plus important que ce qui les oppose au sein du même groupe: ils savent à peu près la même chose et se distinguent peu par leur pratique linguistique: un pacha parle ou (et) écrit en moyenne deux langues. Comme dans la plupart des cas, l'une de ces langues est «maternelle», il n'apprend en moyenne qu'une autre langue, d'expression écrite ou parlée.

L'enquête s'achève sur une reconstitution des âges de la vie (de la période de formation au retrait du service de l'État), au travers des pratiques administratives et des modalités de la carrière. Les pachas débutent généralement leur trajectoire comme jeune surnuméraire dans un bureau de l'État, autant à Istanbul que dans les provinces. Pour s'élever jusqu'au titre, ils ne laissent rien au hasard, cultivent toutes sortes de protections et mobilisent force solidarités familiales. Ils sont formés par des patrons, conseillés par des proches, pilotés par des parents. Hommes de l'épée qui fréquentent les états-majors et partent en mission ou hommes de divan qui administrent l'Empire, ils savent prendre la plume pour solliciter une place, flatter un influent, remercier leur sultan. Véritables marcheurs d'empire dans un espace maîtrisé et traversé plutôt qu'agents instables d'un empire agité par la seule inconstance décisionnelle du Palais, ils vivent une carrière souvent longue de plusieurs décennies, toujours pleinement associée à la vie administrative et politique de l'Empire. Évalués mais rarement sanctionnés, ils participent à l'élaboration de la représentation idéalisée que les agents de l'État se font d'eux-mêmes et de leurs semblables: ce que j'identifie au sein de la culture administrative ottomane comme la figure du «parfait pacha» évoque un agent en fonction avant toutes choses, un acteur éclairé et pragmatique plutôt qu'un honnête homme cultivé, un dignitaire engagé dans des réalisations effectives davantage qu'un Ottoman soucieux de correspondre à la défense de valeurs collectives. Ce que recherche (et valorise) l'administration, c'est un exécu- 
tant efficace, un employé consciencieux et zélé plus qu'un brillant fonctionnaire.

L'ensemble de la recherche permet de montrer que, en l'espace d'un demi-siècle, les pachas ne sont pas devenus les champions de pratiques résolument nouvelles ou les leaders d'une fonction civile et militaire moderne et rationalisée; qu'ils ne sont pas davantage les tenants d'un «traditionalisme» anti-occidental destiné à enterrer la réforme, à ruiner les finances publiques et à corrompre les cadres de l'État. Elle mène plutôt aux conclusions suivantes: même si les pachas ont intégré de nouvelles habitudes et ont renouvelé, auprès de leurs interlocuteurs étrangers, le désir de se placer résolument à l'école européenne, ils veillent à conserver, dans leurs usages et leurs mœurs, l'esprit des institutions plus qu'ils ne cherchent à fonder l'administration de la Turquie moderne. Cadres d'un ancien régime plus que d'un système hamidien ou d'une expérience des Tanzimat, ils sont les serviteurs d'un Empire qui cherche la voie du salut et non les acteurs d'une prétendue modernité en marche. Bref, hommes du XIX ${ }^{\mathrm{e}}$ siècle plus que fonctionnaires en germe du $\mathrm{XX}^{\mathrm{e}}$ siècle, ces piliers d'un État qui continue d'exister après six siècles d'histoire sont encore les pachas du sultan. 